\title{
Proposed atomic cascade experimental test of symmetric local hidden-variables theories
}

\author{
E. S. Corchero \\ Departamento de Física Teórica, Universidad de Cantabria, 39005 Santander, Spain
}

(Received 14 October 1986; revised manuscript received 22 January 1987)

\begin{abstract}
The proposal is made of replacing the usual auxiliary assumptions needed for the derivation of testable Bell inequalities by an assumption resting only on symmetry considerations. It is shown that reliable atomic cascade tests of symmetric local hidden-variables theories should combine high statistics, cascades of type $0 \rightarrow 1 \rightarrow 0$, and calcite polarizers. The three conditions have been achieved in experiments already performed, but never before simultaneously.
\end{abstract}

Several tests of Bell's inequalities by measuring the correlation in the polarization of optical photon pairs emitted in atomic cascases have been performed in the last decade. ${ }^{1-3}$ The experiments have confirmed, in general, the predictions of quantum mechanics and have provided strong evidence against a wide class of local hiddenvariables (LHV) theories. It is well known, however, that a loophole remains for the refutation of the entire family of LHV theories, due to the low efficiency of the available optical photon detectors. ${ }^{4,5}$ Because of this fact, it is necessary to introduce auxiliary assumptions for the derivation of testable inequalities. The purpose of this note is to derive new inequalities, easily testable, using a different auxiliary assumption resting only on symmetry considerations. The possibility of these tests derive from Caser's theorem ${ }^{6}$ (see below). An attempt in the same direction has been made by Marshall, ${ }^{7}$ which concluded that symmetric LHV theories could be tested only with experiments having much better statistics than those so far performed. I shall show that lower statistics is enough, provided that the experiment is made in the appropriate conditions.

The relevant quantity measured in most atomic cascade experiments performed until now is $R(\phi)$, the coincidence counting rate when two polarization analyzers are inserted (one for each photon of a pair) at a relative angle $\phi$. The prediction of quantum mechanics for that quantity is ${ }^{1}$

$$
\begin{aligned}
& R(\phi)=\text { const } \times(1 \pm f \cos 2 \phi), \\
& f \equiv\left[\left(\epsilon_{M}^{1}-\epsilon_{m}^{1}\right)\left(\epsilon_{M}^{2}-\epsilon_{m}^{2}\right) /\left(\epsilon_{M}^{1}+\epsilon_{m}^{1}\right)\left(\epsilon_{M}^{2}+\epsilon_{m}^{2}\right)\right] F(\xi),
\end{aligned}
$$

where $\epsilon_{M}^{1}\left(\epsilon_{M}^{2}\right)$ and $\epsilon_{m}^{1}\left(\epsilon_{m}^{2}\right)$ are the measurable efficiencies of the first (second) analyzer for light with a polarization plane parallel and perpendicular, respectively, to the corresponding analyzer. $F(\xi)$ is a depolarizing factor, slightly smaller than 1 , dependent on the half-angle $\xi$ of the cone of light collected by the lens system. The sign $+(-)$ corresponds to an atomic cascade $J=0 \rightarrow 1 \rightarrow 0$ $(J=1 \rightarrow 1 \rightarrow 0)$.

According to Bell, ${ }^{1}$ the prediction of any LHV theory should be obtained from an expression of the form

$$
\begin{array}{r}
R(b-a)=\mathrm{const} \times \int_{\Lambda} P_{1}(a, \lambda) P_{2}(b, \lambda) \rho(\lambda) d \lambda, \\
0 \leq P_{1}, P_{2} \leq 1,
\end{array}
$$

where $\lambda$ stands for a set of (hidden) variables labeling the state of the photon pair, $\rho(\lambda)$ being the probability density of these states. [Note that a pure quantum state corresponds to an ensemble of given $\rho(\lambda)$ in a LHV theory.] The parameter $a(b)$ labels the angle of the polarization axis of the first (second) analyzer with, say, the vertical plane $(\alpha-b=\phi)$. Clauser and Horne ${ }^{4}$ showed that (1) can be obtained from (2) provided a suitable choice of functions $P_{1}$ and $P_{2}$ is made. Caser ${ }^{6}$ proved later that this is not possible if the functional forms of $P_{1}$ and $P_{2}$ are identical, this being a symmetry condition which corresponds to the approximate symmetry between the two arms of the apparatus used in the experiments. Unfortunately Caser's theorem does not provide any bound for the deviation between the quantum prediction and the prediction of symmetric LHV theories. In the following it is shown that the difference allows a relatively easy empirical test.

The question whether new experiments are necessary deserves a few comments. Most physicists share the opinion that the results of the performed experiments are sufficient, in practice, to refute all local hidden-variable theories although, as a matter of principle, a loophole remains due to the low efficiency of photon detectors. This state of opinion rests upon the belief that, as sometimes stated, the ensemble of photons actually detected is a representative sample of all photons emitted. However, this assumption is not consistent with the fact that even quantum mechanics predicts a nonlinear behavior of photon detectors in some cases. For instance, a light signal corresponding to a two-photon state gives rise to the activation of the detector with a probability $2 \eta-\eta^{2}$, if $\eta$ is the efficiency parameter. Then, let us consider a linearly polarized two-photon signal crossing a polarizer at angle $\theta$. The creation operator for the incoming photons transforms as

$$
a^{\dagger} \rightarrow a_{o}^{\dagger} \cos \theta+a_{e}^{\dagger} \sin \theta
$$

where $a_{o}^{\dagger}\left(a_{e}^{\dagger}\right)$ is the creation operator for photons in the ordinary (extraordinary) ray of the polarizer. Thus, the state with two identical photons transforms as

$$
\begin{aligned}
|2\rangle= & 2^{-1 / 2} a^{\dagger 2}|0\rangle \\
\rightarrow & \cos ^{2} \theta|2\rangle|0\rangle+\sqrt{2} \cos \theta \sin \theta|1\rangle|1\rangle \\
& +\sin ^{2} \theta|0\rangle|2\rangle,
\end{aligned}
$$


where $|2\rangle|0\rangle$ means 2 photons in the ordinary ray and 0 in the extraordinary one, etc. If we consider a onechannel polarizer, the extraordinary ray is absorbed and a detector in the ordinary ray will be activated with a probability

$$
\begin{aligned}
P(\theta) & =\left(2 \eta-\eta^{2}\right) \cos ^{4} \theta+2 \eta \cos ^{2} \theta \sin ^{2} \theta \\
& =2 \eta \cos ^{2} \theta-\eta^{2} \cos ^{4} \theta .
\end{aligned}
$$

That is, the Malus law is satisfied in a measurement with a low-efficiency detector but it is not so if a highefficiency one is used. This illustrates the danger of making extrapolations. In particular, there may be LHV models with $P_{1}(a, \lambda)$ and $P_{2}(b, \lambda)$ nonlinear functions of the detector efficiencies, in such a way that Eq. (2) gives predictions close to the quantum ones in the tested domain, but violating the Bell inequality in experiments, not yet made, with high efficiency detectors.

In this paper, in order to clearly define the problem, I shall call symmetric LHV theories the ones predicting the coincidence counting rate $R(\phi)$ in the form

$$
R(\phi) \propto \int_{c}^{\pi} P(\theta) P(\theta+\phi) d \theta, \quad P(\theta) \geq 0 .
$$

This definition restricts (2) in the sense that here it is considered a single angular hidden variable $\lambda$ and it is assumed that $P$ depends only on the difference $a-\lambda=\theta$ (with $b-a=\phi$ ). If additional hidden variables are included, it is possible to define other forms of symmetric LHV theories able to reproduce the quantum prediction. For instance we may write, instead of (3),

$$
\begin{aligned}
& R(\phi) \propto \int_{0}^{\pi}\left[P_{1}(\theta) P_{2}(\theta+\phi)+P_{1}(\theta+\phi) P_{2}(\theta)\right] d \theta, \\
& P_{1}, P_{2} \geq 0
\end{aligned}
$$

which certainly allows derivation of (1) as easily as (2) does. However, the stronger symmetry condition (3) seems more natural because (4) implies the existence of two sharply different kinds of light signals (for a given wave vector and polarization). Therefore, the symmetric LHV theories defined by (3) appear as a quite natural family that deserves to be, but has not yet been, tested against quantum mechanics.

Tests of symmetric LHV theories have the advantage that the departure between these theories and quantum mechanics is exhibited already by the coincidence counting rate $R(\phi)$ (with both polarizers inserted). In contrast, the inequalities derived with the usual additional hypotheses (e.g., no enhancement ${ }^{4}$ ) involve both $R(\phi)$ and $R_{0}$ (the coincidence rate with the polarizers removed). In consequence, the experimental test of symmetric LHV theories is, in principle, more simple than the tests via Bell's inequalities. Furthermore, those tests are insensitive to detector efficiencies.

In order to study how far the predictions of symmetric LHV theories lie from those of quantum mechanics, we must find an adequate criterion of proximity. To do that, let us assume that an experiment is performed for the test of the quantum predictions, by measuring the number of coincidence counts at several angles, $\left\{\phi_{j}\right\}$, during a given time interval $T$ (the same for all $\phi_{j}$ ) and that results $\left\{N_{i}\right\}$ are obtained. A $\chi^{2}$ test applied to these results will consist of calculating

$$
X \equiv \sum_{j=1}^{n}\left(N_{j}-N_{j}^{Q}\right)^{2} / N_{j}^{Q} \simeq \sum_{j=1}^{n}\left(R_{j}-R_{j}^{Q}\right)^{2} / \delta R_{j}{ }^{2},
$$

where $R_{j}=N_{j} / T\left(R_{j}^{Q}=N_{j}^{Q} / T\right)$ is the measured (predicted) counting rate at the angle $\phi_{j}$, and $\delta R_{j}$ is the error in the measurement of $R_{j}$. The second equality (5) comes from the fact that the predicted standard deviation of the number of counts is $\sqrt{N_{j}}$ if we assume that they follow a Poisson distribution. (We also assume that $\delta R_{j} \ll R_{j}$ and that $N_{j}^{Q}$ is so large that it is possible to approximate the Poisson distribution by a Gaussian, which is needed to give a sense to the $\chi^{2}$ test.) This suggests the definition of the quantity

$$
S=\min _{K \in \mathbb{R}}\left[\sum_{j}\left[\left(K R_{j}^{s}-R_{j}^{Q}\right)^{2} / R_{j}^{Q}\right] / \sum_{j} R_{j}^{Q}\right],
$$

with $R^{s}$ given by (4), as a suitable measure of the "distance" between a given symmetric LHV theory [characterized by a function $P(\theta)$ ] and quantum mechanics. The scale factor $K$ is included in order to emphasize that the normalization of $\left.R^{Q_{(}} \phi_{j}\right)$ is arbitrary. (The main difference between the present work and that of Marshall ${ }^{7}$ lies in that he defined the distance by the mean-square deviation between $R^{s}\left(\phi_{j}\right)$ and $\left.R^{Q_{(}} \phi_{j}\right)$, while here a weighting factor $\left[R^{Q_{(}}\left(\phi_{j}\right)\right]^{-1}$ is included.)

The possibility of an empirical test rests upon the fact that (6) has a positive lower limit $S_{0}$ for some sets of angles $\left\{\phi_{j}\right\}$. It is easy to show that two angles are not enough to discriminate between (1) and (3) and three angles are also insufficient. I have checked that four angles are enough, but the total statistics needed decreases slowly with increasing number of angles, so that five angles seems the optimum number under experimental restrictions. I choose the set $\{0, \pi / 8, \pi / 4,3 \pi / 8, \pi / 2\}$, which has the advantage of including those most frequently measured $^{1,2}$ (Marshall $^{7}$ also chose this set). Then, $S_{0}$ is defined as the minimum of (6) when $R^{s}(\phi)$ is obtained from (3) with $P(\theta)$ any non-negative function satisfying the obvious conditions

$$
P(\theta)=P(-\theta), \quad P(\theta)=P(\theta+\pi) .
$$

A numerical minimization of (6) has been performed and the results are shown in Fig. 1 as a function of the parameter $f$ defined in (1). The numerical calculation of $S_{0}$, for a given $f$, has been made using 17 values of $P(\theta)$ (for $\theta=n \pi / 32$ with $n=0,1, \ldots, 16)$ and then calculating $R^{s}\left(\phi_{j}\right)$ through (3) performing the integral by Simpson's rule [the equalities (7) were used when needed]. The definition (6) was replaced by the equivalent one

$$
S=1-\left[\sum_{j} R_{j}^{s}\right]^{2}\left(\sum_{j}\left(R_{j}^{s}\right)^{2} / R_{j}^{Q}\right]^{-1}\left(\sum_{j} R_{j}^{Q}\right]^{-1}
$$

in order to avoid the unspecified constant $K$. The starting set of values for $P(\theta)$ was chosen from an analytical function suggested by the work of Marshall. ${ }^{7}$ In each step of the minimization, the values of $P(\theta)$ were slightly changed in the direction of steepest descent of $S_{0}$ main- 
taining the condition $P(\theta) \geq 0$. The convergence was good in general, but not always. Sometimes it was necessary to start again with a different initial set of values of $P(\theta)$.

The (non-negative) function $S_{0}$ has a maximum of about $2 \times 10^{-3}$ at $f=1$ and decreases with $f$, being zero for $f \leq 0.5$. The form of the function shows that a test is easier if $f$ is close to 1 . In the experiments so far performed $^{1-3} f$ ranges between 0.84 and 0.96 .

The experimental tests of symmetric LHV theories should discriminate between the hypotheses $S=0$ (quantum prediction) and $S \geq S_{0}$ (symmetric LHV theoretic prediction) by establishing a confidence interval of $S$ from the empirical data. Let us assume that an experiment is performed by recording the number of coincidence counts with the polarizers at the five angles as stated above, the duration of the measurements being the same at all angles. It is possible then to assume that the empirical results $\left\{R_{j}\right\}$ form a sample of a five-dimensional population with mean $\left\{\bar{R}_{j}\right\}$ and standard deviation $\left\{\delta R_{j}\right\}$. Hence, it is necessary the find upper and lower limits of $S$, compatible with this assumption. To do that, from the mathematical equality (for all $j$ )

$$
\begin{aligned}
\left(R_{j}^{Q}-\bar{R}_{j}\right)^{2}+\left(\bar{R}_{j}-R_{j}\right)^{2}-\left(R_{j}-R_{j}^{Q}\right)^{2} & \\
& =2\left(\bar{R}_{j}-R_{j}\right)\left(\bar{R}_{j}-R_{j}^{Q}\right),
\end{aligned}
$$

we obtain

$\frac{1}{2}(S N+Y-X)=\sum_{j}\left[\left(\bar{R}_{j}-R_{j}\right)\left(\bar{R}_{j}-R_{j}^{Q}\right) / \delta R_{j}{ }^{2}\right]$,

where $X$ is given by (5), and I define [compare with (6)]

$$
\begin{aligned}
S & \equiv \sum_{j}\left[\left(R_{j}^{Q}-\bar{R}_{j}\right)^{2} / R_{j}^{Q}\right] / \sum_{j} R_{j}^{Q}, \\
Y & \equiv \sum_{j}\left[\left(R_{j}-\bar{R}_{j}\right)^{2} / \delta R_{j}{ }^{2}\right], \\
N & \equiv S^{-1} \sum_{j}\left[\left(R_{j}^{Q}-\bar{R}_{j}\right)^{2} / \delta R_{j}{ }^{2}\right] \\
& \simeq \sum_{j} N_{j} \simeq \sum_{j}\left({R_{j}}^{2} / \delta R_{j}{ }^{2}\right),
\end{aligned}
$$

so that $N$, as well as $X$, can be calculated from the data. As $\left(R_{j}-\bar{R}_{j}\right) / \delta R_{j}$ is normally distributed, $Y$ has a $\chi^{2}$ distribution, so that $\left\{\bar{R}_{j}\right\}$ must satisfy

$$
0 \leq Y \leq \chi_{M}^{2}
$$

where $\chi_{M}{ }^{2}$ corresponds to the desired confidence interval. For instance, for a $95 \%$ significance level, $\chi_{M}{ }^{2}=11.1$. Then, the confidence interval is given by the set of values of $S$ compatible with (8) and (10). The extreme values of the right-hand side of (8) are found from the Schwarz inequality, which gives

$$
|Y-X+S N| \leq 2 \sqrt{S N Y} .
$$

This leads to the desired upper limit

$$
S_{M}=\frac{1}{N}\left(\sqrt{X}+\sqrt{\chi_{M}^{2}}\right)^{2},
$$

and lower limit

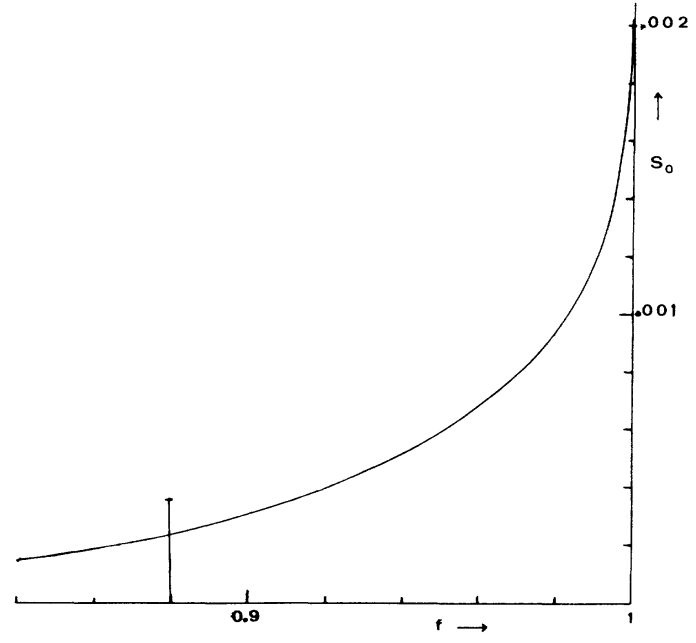

FIG. 1. The "distance" $S_{0}$ between symmetric LHV theories and quantum mechanics as a function of the parameter $f$. At $f \simeq 0.88$ the $95 \%$ confidence interval of $S$ is shown corresponding to the first Orsay experiment (Ref. 2).

$$
\begin{aligned}
& S_{m}=0 \text { if } X \leq \chi_{M}{ }^{2}, \\
& S_{m}=\frac{1}{N}\left(\sqrt{X}-\sqrt{\chi_{M}^{2}}\right)^{2} \text { if } X \geq \chi_{M}{ }^{2},
\end{aligned}
$$

of the confidence interval $\left[S_{m}, S_{M}\right]$. If $S_{m} \neq 0$ there is a violation of quantum mechanics, if $S_{M}<S_{0}$ the symmetric LHV theories are disproved.

A rigorous analysis of the performed experiments is not possible because they have not been made according to the stipulations stated above. In particular, the empirical errors $\delta R_{j}^{2}$ should be proportional to the predicted counting rates $R_{j}^{Q}$. However, we may make the reasonable assumption that the quantities $X$ and $N$ can be calculated by (5) and (9) from the available data. Then, it is easy to see that only the experiments by Aspect et al. ${ }^{2}$ have statistics of the order needed for a test of symmetric LHV theories. In fact, for experiments not violating the quantum prediction (all but one ${ }^{1}$ ), Eq. (11) shows that the relative errors in the measurements should be at most of the order

$$
\begin{aligned}
\delta R / R \simeq 1 / \sqrt{N} & \simeq \sqrt{S_{0} / \chi_{M}^{2}} \\
& \simeq(3-7) \times 10^{-3},
\end{aligned}
$$

while typical values are almost 10 times larger. The Aspect series of experiments have far better statistics, but it is very difficult to estimate $X$ and $N$ from the published data. Only one detailed table of an experimental run has been published, ${ }^{8}$ from which data we have calculated the confidence interval shown in Fig. 1. The experiment is, therefore, compatible with both quantum mechanics and symmetric LHV theories. (It is possible, however, that a detailed analysis of the unpublished data shows a violation of symmetric LHV theories.)

A problem with the Aspect series of experiments is that they achieve a high statistics by using intense atomic sources, which gives rise to a high rate of accidental coin- 
cidences. Although the correction of these by background subtraction is a quite standard procedure, ${ }^{1,2}$ it has been criticized. ${ }^{9}$ In consequence, future experiments should try to avoid any background subtraction.

In summary, the test of symmetric LHV theories requires new experiments combining (1) an atomic cascade of type $J=0 \rightarrow 1 \rightarrow 0$, which allows a factor $F(\xi)$ of about 0.99 , although this (rather geometrical) correction could be confidently included in the LHV prediction and it is, therefore, less relevant, (2) (calcite) polarizers having $\epsilon_{m} / \epsilon_{M}<10^{-4}$, so giving a value greater than 0.999 for the first factor of $f(1)$, and (3) relatively high statistics. The three conditions have been achieved in experiments already performed, but never simultaneously. In particular,
Aspect et $a .^{2}$ failed in the second requirement. On the other hand, calcite polarizers have been used only once, in the experiment by Holt and Pipkin, ${ }^{1,10}$ but the atomic cascade of that experiment was of the type $J=1 \rightarrow 1 \rightarrow 0$, which gave a relatively low value for $F(\xi)$ (about 0.95 ) and, in addition, this experiment has been strongly criticized, ${ }^{1}$ the quantum violation reported in it being currently attributed to systematic errors. It must be taken into account, however, that according to our calculation the conflict between quantum mechanics and symmetric LHV theories should more likely appear in experiments using calcite polarizers, which strongly suggests the need of new experiments with these polarizers in order to clarify the Holt-Pipkin "anomaly."
1J. F. Clauser and A. Shimony, Rep. Prog. Phys. 41, 1881 (1978).

${ }^{2}$ A. Aspect, P. Grangier, and G. Roger, Phys. Rev. Lett. 47, 460 (1981); 49, 91 (1982); A. Aspect, J. Dalibard, and G. Roger, ibid. 49, 1984 (1982); A. Aspect and P. Grangier, Lett. Nuovo Cimento 43, 345 (1985).

${ }^{3}$ W. Perrie, A. J. Duncan, H. J. Beyer, and H. Kleinpoppen, Phys. Rev. Lett. 54, 1790 (1985).

4J. F. Clauser and M. A. Horne, Phys. Rev. D 10, 526 (1974).
${ }^{5}$ T. W. Marshall, E. Santos, and F. Selleri, Phys. Lett. 98A, 5 (1983).

6S. Caser, Phys. Lett. 102A, 152 (1984).

${ }^{7}$ T. W. Marshall, Phys. Lett. 99A, 163 (1983); 100A, 225 (1984).

${ }^{8}$ A. Aspect, thesis, Université de Paris-Sud, Centre d' Orsay, 1983, p. 289.

${ }^{9}$ E. Santos, in Quantum Uncertainties, edited by W. M. Honig (Plenum, New York, to be published).

${ }^{10}$ F. M. Pipkin, Prog. At. Mol. Phys. 14, 281 (1978). 